\title{
Comparison of Different Methods for Identification of Electromyography Threshold in Resistance Exercise

\section{Abstract}

Objective: This study aimed to identify the Electromyographic Threshold (EMGTh) during the dynamic Resistance Exercise, using visual and mathematical identification techniques.

Methods and Results: We evaluated 14 male subjects $(22.6 \pm 2.1$ years) who accomplished an incremental load protocol $(10-10 \%$ of 1RM) in a half squat guided exercise. The intra-class correlation coefficient (ICC) was used to compare the EMGTh identification methods, in which high reproducibility value was obtained $(0.835)$, confirmed by a moderately strong correlation of 0.721 , in general, and yet a pairwise comparison by the Wilcoxon test, which was not identified any difference between the two methods ( $p>0.05$ ).

Conclusion: Therefore, the findings of this study show that both methods, visual and mathematical, used to identify the EMGTh, find the same results.

\section{Keywords}

Electromyography Threshold, Resistance Exercise; Threshold Transition;

Exercise Prescription.

\section{Introduction}

Resistance exercise (RE) is used as a way to promote positive effects on overall health of men and women, as well as in improving the performance of athletes in many sports [1, 2]. It is essential to include parameters that help to control the many variables present in this type of training for the enhance on the inherent gains in the RE.

The surface electromyography (EMG) is an important technique for the evaluation of localized muscle fatigue, recording the changes in action potentials from the muscle [3]. In this sense, some studies have tried to correlate the EMG with the threshold transition (TT) of the
Pablo Ramon Domingos ${ }^{1,2}$, Raul Canestri Galvino Reis ${ }^{2}$, Hiago Leandro Rodrigues de Souza 2,3,

Yuri de Almeida Costa

Campos ${ }^{1,2}$,

Sandro Fernandes da Silva ${ }^{2}$

1 Laboratory of Motor Evaluation/Federal University of Juiz de Fora, Minas Gerais, Brazil.

2 Studies Research Group in Neuromuscular Responses. Federal University of Lavras, MG, Brazil.

3 Studies Research Group in Human Performance and Sports/Federal University of Triângulo Mineiro, Uberaba, Minas Gerais, Brazil.

Contact information:

Sandro Fernandes da Silva.

Address: Nucleus of Studies on Human Movement. Department of Physical Education, University of Lavras. University Campus, PO Box 3037, ZIP Code 37200000. Lavras/MG. Brasil.

झ sandrofs@def.ufla.br 
metabolic systems [4.5] and the results highlighted the EMG as a reliable method for workload control. The identification of these thresholds, as well as electromyography, may be a strategy used to check the intensities in which the transition occurs among the metabolic systems predominance during the exercises, having access to the source of fatigue in a specific situation [6]. Thus, it becomes possible to have a more accurate control of training variables, given that the EMG displays values of an immediate response to exercise, different from the blood lactate which has a delayed response to the stimulus [7].

Conceptually, the electromyographic threshold (EMGth) can be defined from the point of non-linearity among the electromyographic activation values and the workload, as a result of the load increment [8] internal and/or external. The procedure used to determine the EMGth can be provided through a visual model [9], from the inference of experienced evaluators, or also by using a mathematical model by the use of linear regression for identification [10]. However, when it comes to EMGth most studies have allocated their efforts in checking it just in cyclical activities, making your application limited in the RE.

Aguiar et al [7] conducted a study in order to identify the transition threshold by acquiring electromyographic signal and blood lactate in isometric resistance exercises. But its methodology does not reflect the dynamic conditions practices that are performed in the RE. However, Nasser [11] presented by the visual identification method, for the biceps curl, transition thresholds (TT) by lactacidemy, spirometry and the EMG, through the signal conduction velocity obtained, and found that EMGTh is equivalent to the gold method for identification of TT, lactacidemy in upper limbs in single-joint exercise. Thus, the aim of this study was to identify the EMGth in ER multiple joint and lower limbs with recreational athletes, using the different techniques, visual and mathematical identifications.

\section{Methods}

\section{Subjects}

The sample consisted of 14 male subjects (22.6 \pm 2.1 years, $178.9 \pm 4.7 \mathrm{~cm}, 81.5 \pm 10.4 \mathrm{~kg}$ and 19.9 $\pm 5.7 \%$ fat) all college team sports athletes (rugby, basketball, handball, futsal) healthy, and with experience of at least 6 months of strength training and without musculoskeletal injuries. The project was approved by the Ethics Committee of the Universidade Federal de Lavras with the protocol number of CAAE: 31822714.6.0000.5148.

\section{Procedures}

At first, the evaluation to characterize the sample was carried out, and, soon after, a test of maximum repetition (1RM) [12] for the guided half squat (Physicus ${ }^{\circledR}$ ). After an interval of 72 hours, the subjects performed the protocol of progressive loads for the guided half squat. For this procedure were made progressive increases of 10 to $10 \%$ of $1 \mathrm{RM}$ workload until the value of $70 \%$. Each exercise series lasted for one minute, it was defined 2 seconds for each phase of the dynamic motion (eccentric and concentric) controlled by a digital metronome (Metronome Plus ${ }^{\circledR}$ ). Between each series, it was observed a recovery range equivalent to two minutes. The exercise was stopped at the time that the subjects did not undergo the pace pre-established, resulting of the maximal voluntary fatigue for that situation, or when the subject asked to stop, or if reached $70 \%$ of 1 RM. Electromyography was recorded through a Miotool electromyography, 400 Miotec ${ }^{\circledR}$ model. It was used a SDS500 sensor of gain of 1000 times and $2000 \mathrm{~Hz}$ of sampling frequency. Subsequently, the signal was subjected to a section, excluding the first and last repetition and further subjected to a bandpass filter between 20 to $500 \mathrm{~Hz}$, using for the analysis the values of root mean square (RMS). The muscles analyzed were Rectus Femoris (RF), Vastus Medialis (VM) and Vastus Lateralis (VL). 
In order to determine the electromyographic threshold (EMGTh) by the mathematical method, RMS data were used in a simple regression equation and the point of greatest distance below the straight line was determined as the EMGTh [8]. In the visual inspection method, two researchers observed the RMS data and from the break point of linearity of the points was determined EMGTh [13].

\section{Statistical Analyses}

For statistical analysis we used the SPSS software, version 21.0 for Windows. As it presents the statistics by mean \pm standard deviation, normality was tested by the Kolmogorov-Smirnov test, and sphericity by Mauchly test with epsilon's correction of Huynh-Feldt. To compare the results of different EMGTh identification methods was used Intraclass Correlation coefficient, with confirmation by Pearson's correlation.

It was still used the Wilcoxon test for comparison between the different stages in which the thresholds were found for each grouping in Visual and Mathematical method, and for confirmation was used comparison by ANOVA of repeated measures with subsequent paired analysis of Bonferroni, among the values obtained at each stage and the values corrected by simple regression equation. Always adopting the significance value $p<0.05$.

\section{Results}

The intra-class correlation coefficient (ICC) was used to compare the EMGTh identification methods, where high reproducibility value was obtained (0.835), overall confirmed by a moderately strong Pearson's correlation of 0.721 .

Comparison among the pairs of stages which the thresholds of muscles were determined in both methods, there was no significant difference between any of them (VM p = 0.785; RF $p=0.063$; and $V L p=0.317$ ) by Wilcoxon statistical test, as shown in Table 1.

Even so, using ANOVA of repeated measures, the average values of RMS were tested, the collected
Table 1. Comparison of the points identified as EMGTh in Visual and Mathematical methods in load percentage.

\begin{tabular}{|l|c|c|c|}
\multicolumn{1}{|c|}{ Muscles } & Visual & Mathematical & ICC \\
\hline Vastus Medialis (\%) & $38.6 \pm 9.5$ & $37.8 \pm 11.9$ & 0.83 \\
\hline Retus Femoris (\%) & $41.4 \pm 10.3$ & $37.1 \pm 11.4$ & 0.83 \\
\hline Vastus Lateralis (\%) & $40.0 \pm 9.6$ & $36.4 \pm 10.1$ & -0.17 \\
\hline
\end{tabular}

Comparison of the stages in which the visual and mathematical EMGTh were found.

values and the ones corrected by simple regression equation, this way was determined the points where there was the breaking of linearity among the stages, with significant differences for all groups ( $p$ $=0.001$ for VM on the collected data, and for the others $p<0.001$ ), according to Figures 1 and 2 .

Figure 1: Identification of the EMG threshold by the Visual Method.

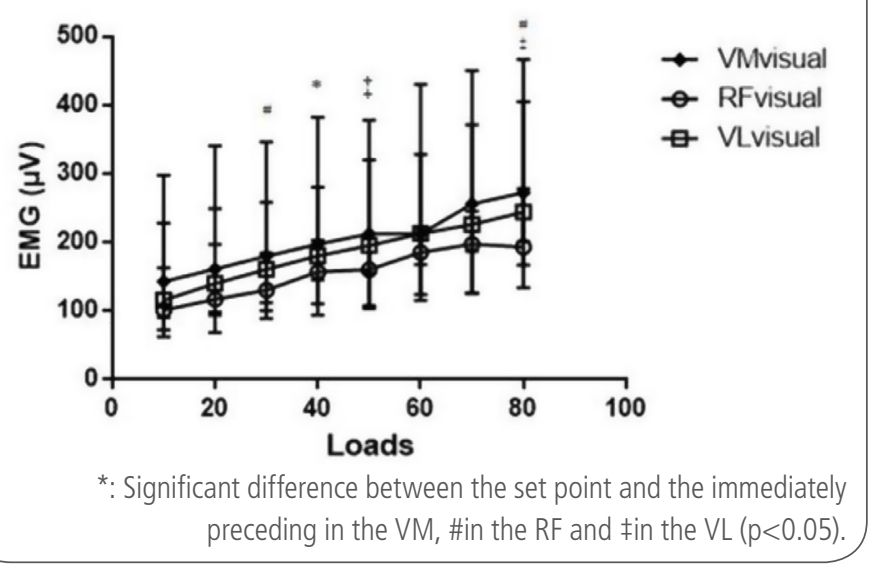

Figure 2: Identification of the EMGTh by the Mathematical Method.

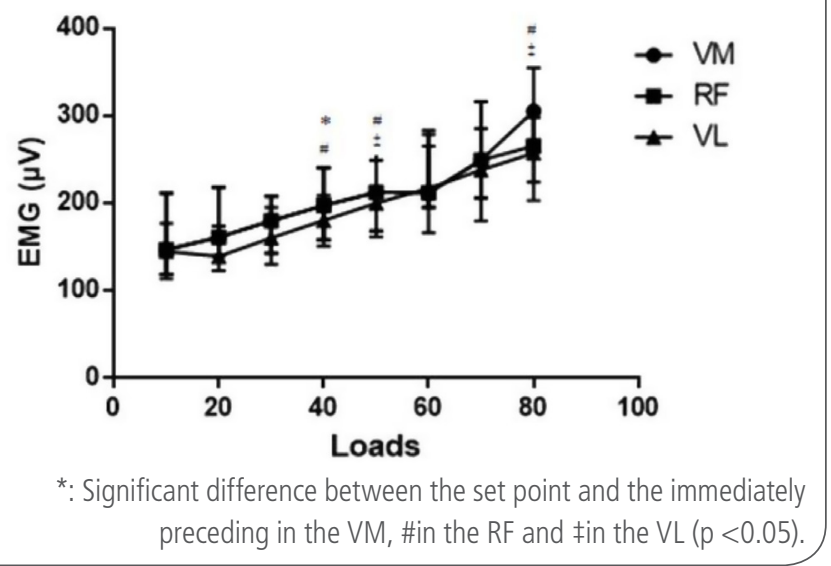




\section{Discussion}

This study aimed to compare two methods for identification of the electromyographic threshold in resisted exercise of the lower limbs, which found no difference in the identification of the same, with high reproducibility in the ICC (0.835), therefore, both methods agree with the point identified as EMGTh.

The results of this study, even for the RE, confirm the findings of Hug et al [14] which showed no significant differences between the two methods by observing the test stage and the RMS of the EMG. Having been presented the ICC for each grouping analyzed a value of good agreement between the two methods of identification, as well as the overall value.

The stages where there was identification of EMGTh corroborate the bibliographic findings which was found around 30 and $40 \%$ of $1 \mathrm{RM}[13,15]$, this values were also found in this study, however, Lucia et al [16] had shown on a progressive test of a cyclic modality the anaerobic threshold at intensities close to 60 to $70 \%$ of $\mathrm{VO}_{2 \text { max }}$ suggesting that the EMGTh is related to the activity type and protocol, yet having a highly reproducible and significant ICC according to Lucia et al [17], the EMGTh shows no significant difference, and shows significant correlation to the lactate and ventilatory thresholds expressed in $\% \mathrm{VO}_{2 \max }$ for heart transplanted.

Therefore, the identification of EMGTh seems to be a good tool for RE prescription, and no significant differences were found among the stages of threshold found in visual methods and mathematical, which confirms the ICC, with also the correlation of 0.721 to all groups in general.

The identification of the EMGTh depends on the composition of the muscle fibers of the grouping analyzed, thus, the VL reaches lower transition threshold values than the other groups, qualitative analysis, as well as the findings of Aguiar et al [7], perhaps due to the fact that VL has a higher percentage of fast fibers [18], besides the composition of muscle fibers of each muscle studied [16]. The mostly dynamic conditions for strength training, antagonize the methodology applied by Aguiar et al [7] due to the principle of specificity, having already been reported a decrease in strength in incremental protocols for isometric RE, but EMGTh also presents sensitive to these changes allowing identification even in different situations.

If there is some difference between the intensity that identifies the EMGTh and lactate Threshold (13 and 16\% of 1RM) Aguiar et al [7] justified by the immediate response obtained in the EMG, unlike blood lactate collection, which would make the EMGTh a more precise tool and objective about the assessment during the exercise, considering that the EMG have access to muscle physiological processes noninvasively, but Lucía et al [16] showed similarity among the identification methods of the transition thresholds, which infers that the EMGTh suffers a strong dependence on the muscle-test ration, being the first held with isometric RE and the second on a ergometer cycle (ramp test).

For the biceps curl, the EMGTh matches the Lactate Thresholds (LT), being the first transition threshold found for Lactate in $21.5 \pm 2.4 \%$ of $1 \mathrm{RM}$ and EMGTh $22.7 \pm 3.3$, but LT2 was determined subsequently to the EMGTh $(32.1 \pm 2.5$ and $28.8 \pm 2.9 \%$ of $1 R M$, respectively) due to mechanical factors of the RE [11], in this study the thresholds of the VM muscles, RF and VL were found in about $40 \%$ of $1 \mathrm{RM}$, however, the load increase was from 10 to $10 \%$ starting at $10 \%$, and Nasser [11] worked with $20 \%$ in increments of 5 to $5 \%$ up to $40 \%$ of 1 RM, and subsequently to that of 10 to $10 \%$, using 3 minutes per stage $(1$ minute work and 2 minutes rest), but with different amounts of movements, 15 and 20 movements, respectively. The EMGTh found in this study suggests that it is the second TT, given that in Nasser's work [11] was determined to $28.8 \%$ of $1 \mathrm{RM}$, which comes to confirm a relationship between LT and the work time, and not necessarily just workloads.

Therefore, the findings of this study show that both methods, visual and mathematical, for identi- 
fication of the EMGTh find the same results. Thus, the use of EMGTh for resistance exercise prescription is a good method because of its convenience, lower cost compared to other methods and is not invasive, besides it yet presents values of immediate response during exercise.

\section{Funding}

This article is the result of a scientific initiation research funded/financed/sponsored by FAPEMIG (Minas Gerais State Support Fund for Research).

\section{References}

1. Garber CE, Blissmer B, Deschenes MR, Franklin BA, Lamonte MJ, Lee I-M, et al. American College of Sports Medicine position stand. Quantity and quality of exercise for developing and maintaining cardiorespiratory, musculoskeletal, and neuromotor fitness in apparently healthy adults: guidance for prescribing exercise. Med Sci Sports Exerc. julho de 2011; 43(7):1334-59.

2. Ribeiro AS, Avelar A, Schoenfeld BJ, Fleck SJ, Souza MF, Padilha $\mathrm{CS}$, et al. Analysis of the training load during a hypertrophy-type resistance training programme in men and women. Eur J Sport Sci. 2015; 15(4):256-64.

3. Crozara LF, Castro A, De Almeida Neto AF, Laroche DP, Cardozo AC, Gonçalves M. Utility of electromyographic fatigue threshold during treadmill running. Muscle Nerve. dezembro de 2015; 52(6):1030-9

4. Candotti CT, Loss JF, Melo M de O, La Torre M, Pasini M, Dutra LA, et al. Comparing the lactate and EMG thresholds of recreational cyclists during incremental pedaling exercise. Can J of Physiology and Pharmacology. maio de 2008; 86(5):272-8

5. Hug F, Laplaud D, Lucia A, Grelot L. EMG threshold determination in eight lower limb muscles during cycling exercise: a pilot study. Int J Sports Med. junho de 2006; 27(6):456-62.

6. Cochrane KC, Housh TJ, Bergstrom HC, Jenkins ND, Johnson GO, Schmidt RJ, et al. Perceptual and Physiological Fatigue Thresholds during Cycle Ergometry. J of Exercise Physiology Online [Internet]. 2014 [said 23 July 2015]; Available em: https:// www.asep.org/asep/asep/JEPonlineOCTOBER2014 Cochrane. $\underline{\mathrm{pdf}}$

7. Aguiar AP de, Oliveira JC de, Stefanelli VC, de Oliveira MG, de Azevedo PHSM, Baldissera $V$, et al. Efeito do incremento de carga na resposta eletromiográfica e no lactato sanguíneo durante exercício estático. Rev Anda de Med del Dep. 2010; 3(2):62-7.

8. Hug F, Laplaud D, Lucia A, Grelot L. EMG Threshold Determination in Eight Lower Limb Muscles During Cycling Exercise: A Pilot Study. Int J Sports Med. junho de 2006; 27(6):456-62.

9. Hug F, Laplaud D, Savin B, Grélot L. Occurrence of electromyographic and ventilatory thresholds in professional road cyclists. Eur J Appl Physiol. novembro de 2003; 90(56):643-6.
10. Lucía A, Vaquero AF, Pérez M, Sánchez O, Sánchez V, Gómez $M A$, et al. Electromyographic response to exercise in cardiac transplant patients: a new method for anaerobic threshold determination? Chest. junho de 1997; 111(6):1571-6.

11. Nasser SV. Identificação dos limiares de lactato, ventilatórios e eletromiográficos de superfície em exercício resistido [Dissertação Dissertation]. [Brasília]: Universidade de Brasília; 2011.

12. Brown LE, Weir JP. Asep Procedures Recommendation I: Accurate Assessment Of Muscular Strength And Power. Professionalization of Exercise Physiology [Internet]. 2001 [said 14 July 2015]; 4(11). Available em: http://search.ebscohost.com/login. aspx?direct=true \&profile=ehost $\&$ scope $=$ site \&authtype $=$ crawle r\&jrnl=10995862\&AN=19994524\&h=b4maFj3PQgKF4ZeSZagl W\%2BVzEz5LdvPoo3B2ZxUlh9N2wrUeGUHRCVv1luPM3367G lkAnBJ9bsLVHmW8ib8VBw\%3D\%3D\&crl=c

13. Hug F, Laplaud D, Lucia A, Grelot L. A Comparison Of Visual And Mathematical Detection Of The Electromyographic Threshold During Incremental Pedaling Exercise: A Pilot Study. Journal of Strength and Conditioning Research. 2006; 20(3):704-8.

14. Hug F, Laplaud D, Mulas AL, Grelot L. A Comparison of Visual and Mathematical Detection of the Electromyographic Threshold During Incremental Pedaling Exercise: A Pilot Study. Journal of strength and conditioning research: the research journal of the NSCA. 2006; 20(3):704-8.

15. Oliveira ASC, Cardozo AC, Barbosa FSS, Gonçalves M. Electromyographic fatigue threshold of the biceps brachii: the effect of endurance time. Electromyogr Clin Neurophysiol. fevereiro de 2007; 47(1):37-42.

16. Lucía A, Sánchez $O$, Carvajal A, Chicharro JL. Analysis of the aerobic-anaerobic transition in elite cyclists during incremental exercise with the use of electromyography. $\mathrm{Br} J$ Sports Med. junho de 1999; 33(3):178-85.

17. Lucía $A$, Vaquero $A F$, Pérez $M$, Sánchez $O$, Sánchez V, Gómez $M A$, et al. Electromyographic response to exercise in cardiac transplant patients: a new method for anaerobic threshold determination? Chest. junho de 1997; 111(6):1571-6.

18. Pierrynowski MR, Morrison JB. A physiological model for the evaluation of muscular forces in human locomotion: theoretical aspects. Mathematical Biosciences. 10 de julho de 1985; 75(1):69-101

Publish in International Archives of Medicine

International Archives of Medicine is an open access journal publishing articles encompassing all aspects of medical science and clinical practice. IAM is considered a megajournal with independent sections on all areas of medicine. IAM is a really international journal with authors and board members from all around the world. The journal is widely indexed and classified Q2 in category Medicine. 